\title{
INTEGRATED CHEMICAL ANALYSIS ON PLASTIC MICROFLUIDIC DEVICES
}

\author{
Travis D. Boone, Herbert H. Hooper, and David S. Soane \\ Soane BioSciences, Inc., Hayward CA 94545-3716
}

\begin{abstract}
Soane BioSciences is developing low-cost, mass-produced, plastic microfluidic devices for a variety of microfluidic applications including DNA analysis, clinical diagnostics, and drug discovery. Plastic substrates with complex patterns of 10-100 $\mu \mathrm{m}$-sized channels are reproducibly formed against mold tools fabricated by micromachining techniques. These substrates are used to produce plastic devices on which high-efficiency electrophoretic separations of DNA and other biomolecules have been achieved in 1-10 minutes. Plastic microfluidic devices offer several advantages over glass or silicon structures including lower processing temperatures, greater surface treatment options for electro-osmotic flow control, lower cost, and simpler extension to multi-layer device fabrication.
\end{abstract}

\section{INTRODUCTION}

The concept of microfabricating one or more components of analytical instruments dates at least to the work of Terry and coworkers, who described a microfabricated gas chromatograph almost 20 years ago [1]. The rationale for employing microfabrication technologies in analytical instrumentation are many: reducing instrument size and cost, reducing sample and reagent volumes, reducing analysis time, increasing analysis throughput, and integrating sample preparation and analysis functions. The specific objectives for a given program depend very much on the application and customer environment.

In the area of liquid-phase analysis, various methods have been explored for pumping and manipulating fluids in microstructures. A particularly promising approach is the use of electrokinetic phenomena, which avoids mechanical pumps and valves. Pace [2] and Kovacs [3] were the first to propose the concept of "chip-CE", wherein capillary electrophoresis separations are performed in microfabricated columns on planar substrates. Subsequently, groups at Ciba-Geigy [4-7], University of Alberta [8,9], Oak Ridge National Laboratory [10,11], University of California Berkeley [12,13], and PerSeptive Biosystems [14] have demonstrated various implementations of electrically-driven separations on microfabricated chip devices. In addition to separations, several groups have explored "integrated" chip-CE, meaning the integration of various analytical protocols (e.g., mixing, reaction, separation) onto a single chip [15-17].

Most micro-scale electrophoretic devices have been fabricated from glass or silica. However, when single-use devices are desired, glass chips may not be cost-effective. As early as 1990, Soane [18] and Eckstrom [19] proposed the use of polymeric materials in chip-CE. Soane focused on thermoplastics such as polymethylmethacrylate (PMMA), while Eckstrom et al. investigated elastomeric polymers such as PDMS. More recently, the Novartis group [20] has demonstrated electrophoretic separations in PDMS microchannel devices.

We have been developing technology for the manufacture and use of polymeric devices in chip-CE applications, with the goal of producing single-use chips at an acceptable cost structure. We have reported results for chip-CE in PMMA microdevices [21] and in composite devices with a PMMA base enclosed by an elastomeric PDMS cover [22]. We have also explored and employed numerous other base and cover materials, and investigated the suitability of their optical, mechanical and surface properties in various applications. In this paper, we report on some of our observations and results.

\section{Plastic Chip Fabrication}

\section{EXPERIMENTAL DETAILS}

There are numerous methods for forming capillary size channels in polymeric substrates. However, several methods, such as direct machining or laser etching, can produce surfaces which are not sufficiently smooth for capillary electrophoresis. Replication methods, such as injection molding, compression molding, and casting tend to provide much smoother surfaces which we have found very suitable for capillary electrophoresis.

One general approach which we have employed involves two primary steps: (1) formation of microstructures in a base layer, and (2) sealing of the base layer with a cover layer. To form the base layer, microfluidic patterns are first micromachined on a glass or silicon master (see Figure 1) using common photolithographic and etching techniques. A metal mold is then formed via electroplating against the master. Such metal molds can be mounted on tooling for injection molding, as shown in Figure 2. Polymer substrates are then formed in large volumes by injection or compression molding (Figure 3). These replicates can be discrete chips, or may consist of multiple chip patterns which can be diced out of a composite part, as shown in Figure 4. Extremely smooth and precise channels of micrometer dimensions can be formed, as demonstrated by the actual 3-D profile of a molded plastic chip in Figure 5.

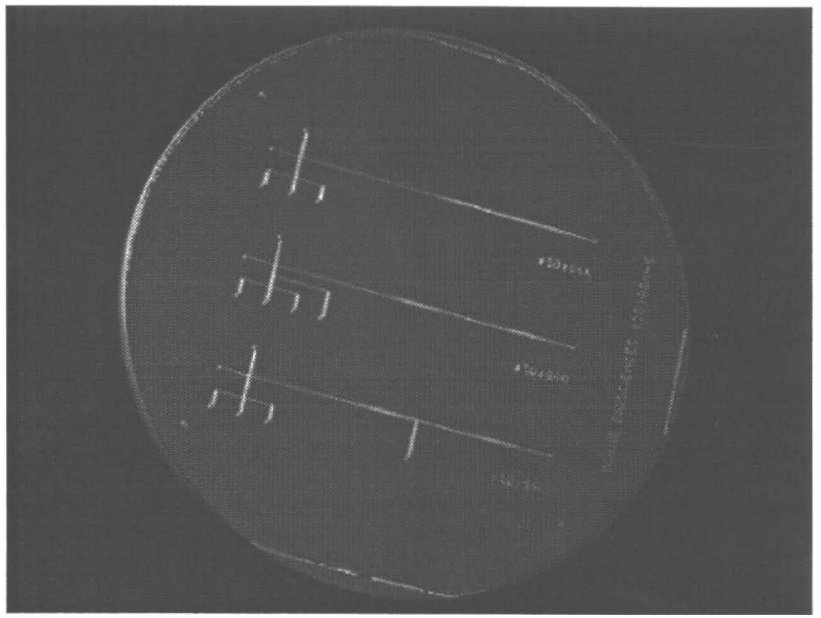

Figure 1. 4" silicon wafer with etched microfluidic patterns. 
Figure 2. Nickel a silicon master and mounted on an injection molding insert. electroform grown on
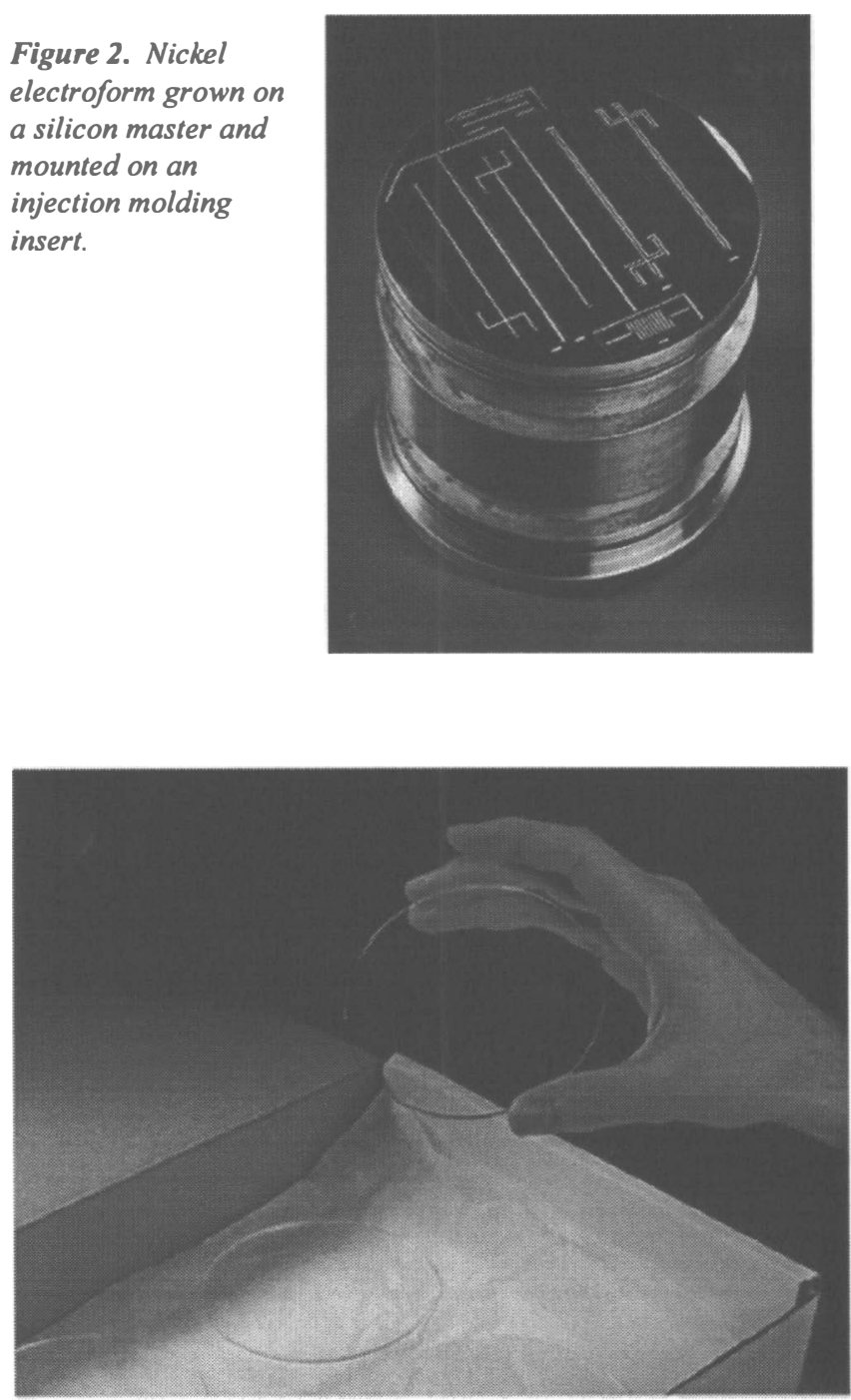

Figure 3. Plastic microfluidic substrates can be mass produced at low cost.

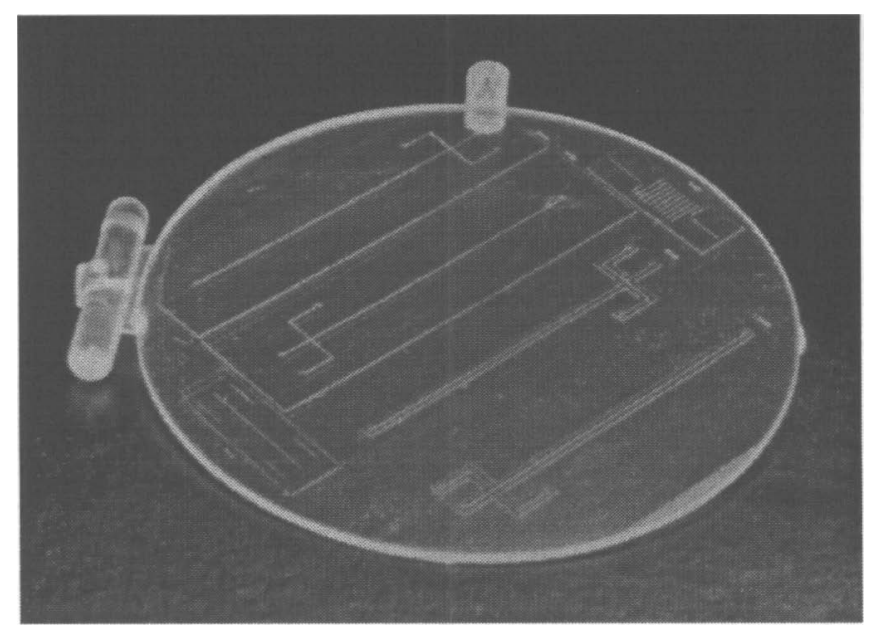

Figure 4. An injection molded plastic microfluidic substrate formed from the mold tool shown in Figure 2.

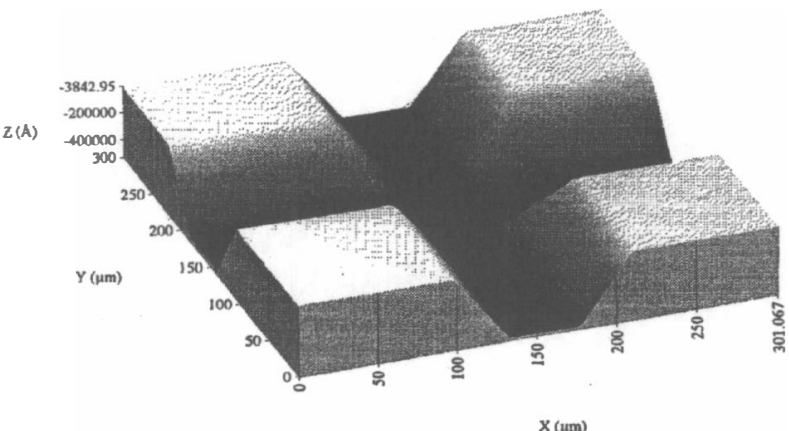

Figure 5. Profilometry scan of an injection molded plastic microfluidic channel intersection. Measurement was performed on a Tencor P-10 system. The channels are approximately $100 \mu \mathrm{m}$ wide and $50 \mu \mathrm{m}$ deep.

We have developed proprietary methods to form enclosed microchannels by sealing various polymer films to base substrates. Figure 6 shows an example of a completed, sealed and functional plastic chip ready for use.

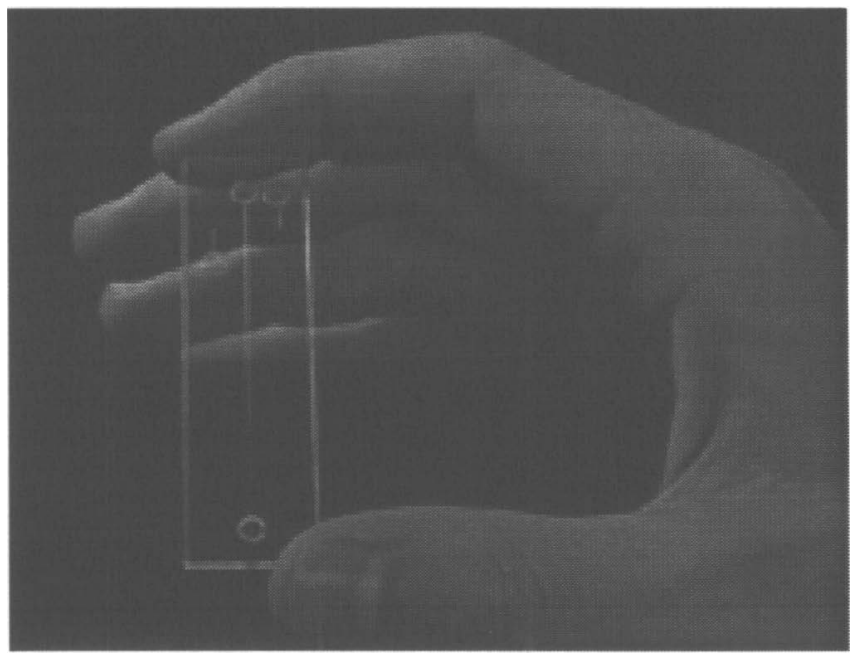

Figure 6. A sealed, functional plastic microfluidic card with a cross channel for nanoliter injections and separations of sample. The channels of $100 \mu \mathrm{m}$ width $\times 50 \mu \mathrm{m}$ depth connect four reservoirs for samples and electrode contacts.

In addition to rigid, injection molded chips (approximately $1 \mathrm{~mm}$ in thickness), we have produced microfluidic structures in flexible film formats $(\sim 100 \mu \mathrm{m}$ thick). An example of a multiplexed microfluidic pattern on a film format is shown in Figure 7. Continuous films with formats which mate to standard microtiter well patterns can be employed to perform biological assays in high throughput. 


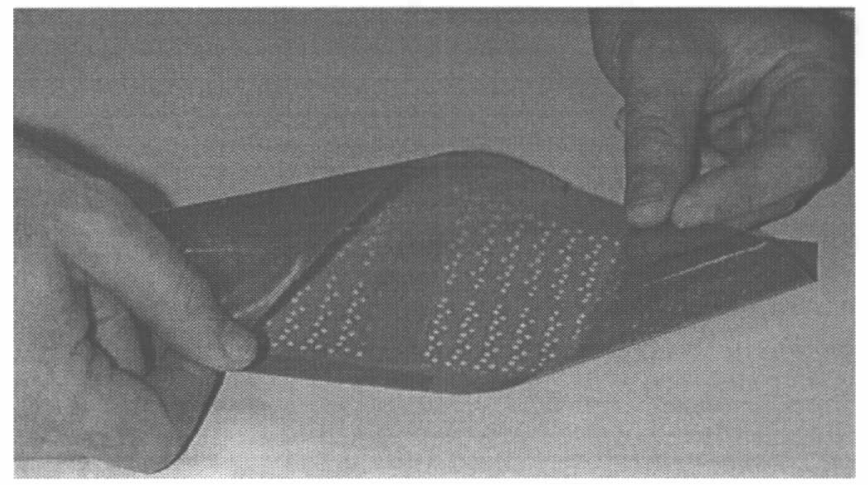

Figure 7. Multiplexed microfuidics in a continuous plastic film. Reagent micro-reservoirs mate with standard microtiter plate formats.

\section{Surface Treatment and Fluid Manipulation}

Electrokinetic flow involves both electrophoretic and electroosmotic components. While one or the other may dominate in a given situation, both forces usually contribute to analyte or reagent movement, as most biomolecules carry a charge, and most surfaces have some degree of charge in aqueous buffers. A great deal is known regarding electro-osmotic flow (EOF) in fused silica capillaries, based on the long history of fused silica columns in capillary electrophoresis, and the numerous methods which have been developed to control EOF in these columns. This knowledge from fused silica columns is directly transferable to glass chips.

Comparatively little is known regarding EOF in plastic capillaries or channels. We have developed surface chemistries and treatment methods which enable us to achieve desired magnitudes of electroosmotic flow in plastic microchannels. The level of electroosmotic forces desired depends on the specific microfluidic manipulations to be performed. EOF should be low for highresolution nucleic acid separations, moderate for stream contacting and mixing, and high for fluid transport and capillary electrochromatography. We have developed various surface treatment techniques to span this desired range of performance, as demonstrated in Figure 8. Lifetime studies indicate that these surface modifications can generate stable electro-osmotic flow rates over long periods and multiple runs.

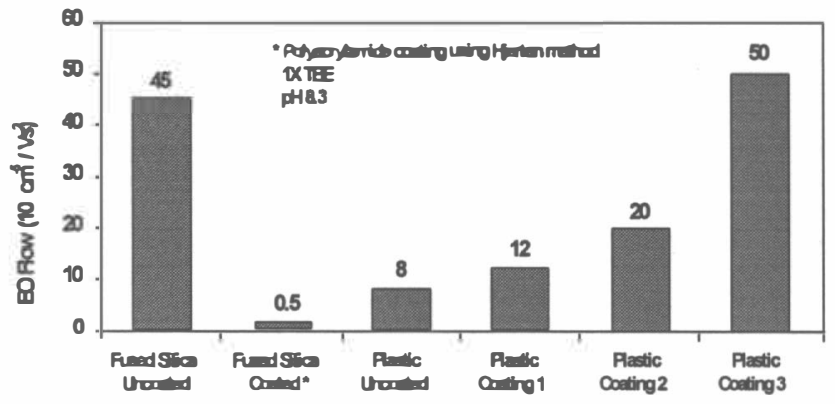

Figure 8. Effect of surface modifications on electro-osmotic flow for fused silica and plastic capillaries.

With controlled surface properties, electro-osmotic flow can be used to meter, dispense and mix fluid streams on plastic devices.
Figure 9 demonstrates our ability to mix two electrokineticallydriven streams at a $T$ junction. The relative amounts of the two streams entering the downstream channel are precisely and independently controlled by the applied electric fields.

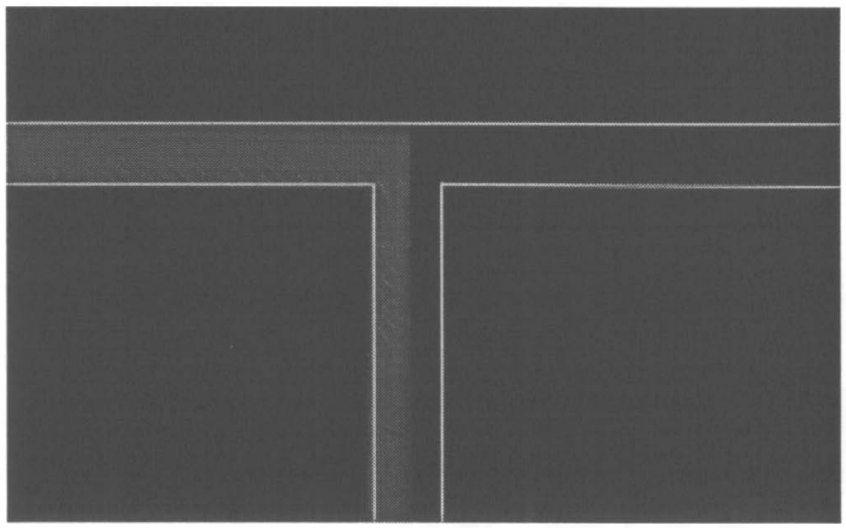

Figure 9. Electrokinetically controlled mixing of a fluorescent and non-fluorescent fluid at a T intersection on a plastic chip. The incoming streams meet head on at the top of the figure, then flow toward the bottom where they mix by diffusion. The ratio of the two incoming flows can be electronically controlled. Channels: $\sim 100 \mu \mathrm{m}$ width, $\sim 35 \mu \mathrm{m}$ depth.

Liquid measurement and dispensing is a ubiquitous operation in biology and analytical labs. In Figure 10 a succession of images is presented showing on-chip liquid measurement and dispensing, wherein a nanoliter size plug is being dispensed into a channel. This capability is important not only for sample and reagent metering and dispensing, but also for sample introduction in onchip separations.
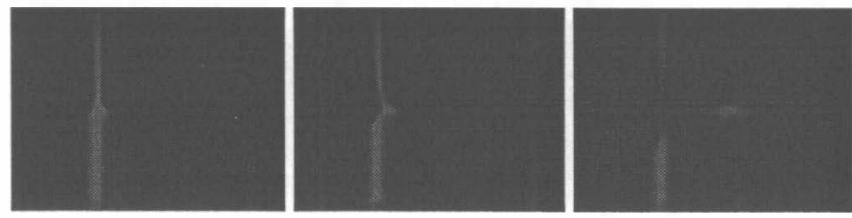

Figure 10. Sub-nanoliter (0.5nl) injections at a cross injector on a plastic chip. A fluorescent dye has been added for visualization.

In order to apply high electric fields for rapid, high-resolution electrophoretic separations, the chip must effectively conduct heat away from the fluid in channels. Figure 11 compares an Ohm's law plot derived on the acrylic chip with a similar plot derived from a conventional fused silica capillary of length dimension identical to that of the acrylic chip channel $(5.5 \mathrm{~cm})$. The plots were derived from a channel or capillary filled with $0.5 \mathrm{X}$ TBE buffer; voltage across the channel/capillary was increased in 200250 volt increments starting at 0 volts, and after a stabilization period of $\sim 15$ seconds, the current through the channel/capillary was recorded. For this experiment, a $75 \mathrm{~mm}$ ID polyimide-clad fused silica capillary was used to derive a comparative Ohm's law plot. Figure 11 reveals that the trend of the plot for the acrylic channel is similar to the plot for the fused silica capillary, with both drawing $\sim 20 \mathrm{~mA}$ current at 5000 Volts applied potential. This similarity implies that the cross-sectional area of the acrylic 
channel and of the silica capillary are essentially identical. Finally, based on the close similarity of the two plots, the heat dissipation properties of the acrylic channel compares favorably to the heat dissipation from the silica capillary, at least at the low power conditions ( $\sim 100$ milliwatts) used here. The circumferential area for a non-circular chip channel is greater than for a circular capillary with equivalent cross-sectional area; thus, a larger circumferential surface area-to-volume ratio could yield enhanced heat dissipation in the chip and could compensate for the poorer thermal conductivity of the plastic chip. The field strength in these experiments intentionally was limited to $\sim 1100 \mathrm{~V} / \mathrm{cm}$, though no problems were experienced with dielectric breakdown of the chip components at the maximum field strength.

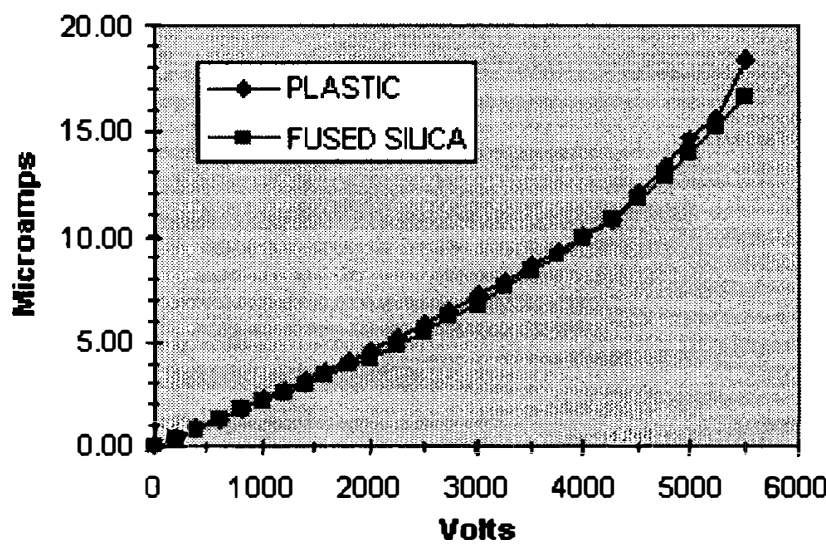

Figure 11. Ohm's law plot comparing the heating behavior of silica capillaries and plastic channels. $5.5 \mathrm{~cm}$ separation lengths were used with 0.5x TBE. Capillaries and chip channels had comparable cross-sectional areas of $\sim 4400 \mu \mathrm{m}^{2}$.

\section{Detection}

Detection is accomplished with a confocal fluorescence approach similar to the work of Mathies et al. [23]. A basic schematic of the breadboard platform is shown in

Figure 12.

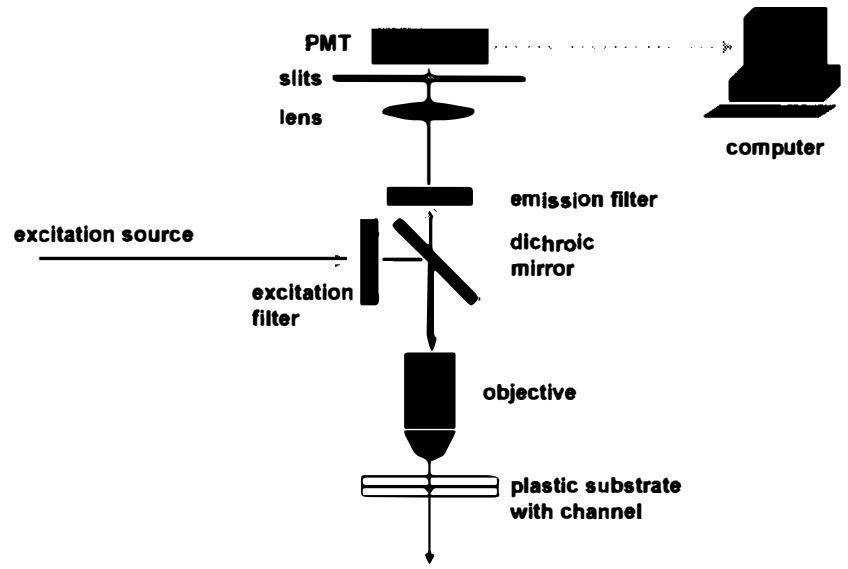

Figure 12. Schematic diagram of confocal laser-induced fluorescence (LIF) detection.

\section{Rapid DNA Separations}

\section{RESULTS}

We have demonstrated rapid, high-resolution separations of nucleic acids in plastic microfluidic devices. An example of a separation of a double-stranded DNA restriction digest is shown in Figure 13. This chip-CE separation is similar in speed and resolution to separations reported with glass devices [12], indicating that plastic devices can provide comparable performance to glass.

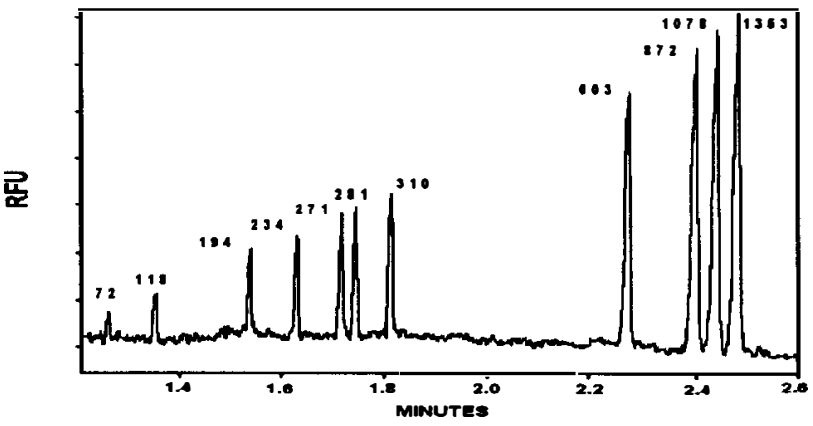

Figure 13. Separation of Hae III $\phi X 174$ on a plastic chip. Buffer: $0.5 x T B E, 0.5 \%$ polymer gel, $2.5 \mu \mathrm{g} / \mathrm{ml} \mathrm{EtBr}$. Conditions: $180 \mathrm{~V} / \mathrm{cm}$, detection $4 \mathrm{~cm}$ from injection, Ex: $488 \mathrm{~nm}$.

Table 1 summarizes run-to-run and chip-to-chip reproducibility data for migration times for the 11 peaks in the Hae III digest of $\phi X 174$ DNA on 7 chips randomly selected from an injection molding run of 500 chips. The relative standard deviation (\%) is shown for six sequential runs on each chip as well as composite reproducibility for all data on each peak on all chips. The run-torun migration time reproducibility is quite good, with RSD values below $1 \%$ for all peaks. No effort was made to control the chip temperature during the course of these runs and thus some variation in migration times due to changes in room temperature would be expected. Chip-to-chip reproducibility of migration times was somewhat higher than run-to-run reproducibility and ranged from 2.0 to 3.0 percent among the 7 chips used in this evaluation. This may be explained by slight variations in the effective field strengths and effective migration distances used in the evaluation among the 7 chips since a constant voltage was used for all chips and the total channel length varied by about $1.5 \%$.

\begin{tabular}{llllllclllll}
\multicolumn{11}{c}{ Peak } \\
\cline { 2 - 7 } & 1 & 2 & 3 & 4 & 5 & 6 & 7 & 8 & 9 & 10 & 11
\end{tabular}

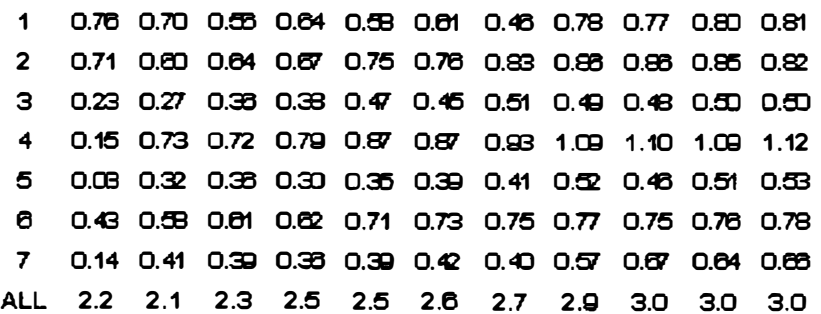

Table 1. RSD \% of the migration times of Hae III $\phi X 174$ peaks from runs of the type shown in Figure 13. 
Separations of sequencing reactions have also been performed on plastic chips, as shown in Figure 14. Note that single base resolution is being approached for up to 350 bases in only 10 minutes.

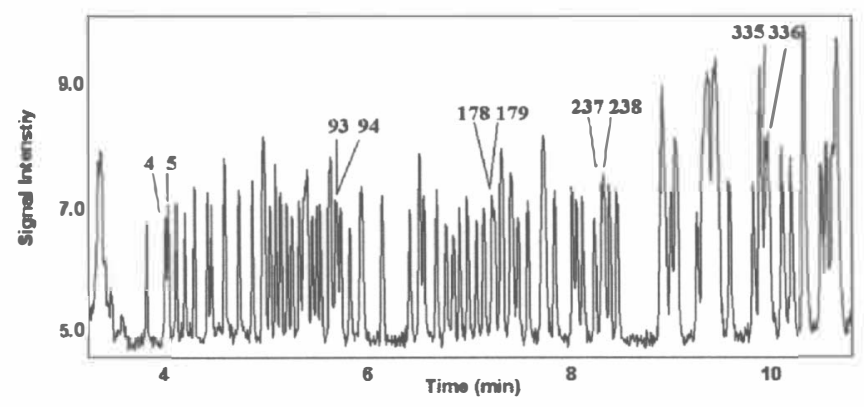

Figure 14. Rapid, high-resolution separation of ssDNA in a plastic chip. M13mpl8 T-terminated sequencelenergy-transfer (FAM-TAMRA) primer, 5\% uncrosslinked polymer, 7M Urea, $1 X$ TBE, $200 \mathrm{~V} / \mathrm{cm}$, Ex: Ar laser at $488 \mathrm{~nm}, \mathrm{Em}: 570-590 \mathrm{~nm}$.

We have also performed competitive immunoassays and enzyme inhibition assays in plastic microfluidic devices. Competitive binding assays with electrophoretic separation of bound and free human insulin have been performed with nanomolar sensitivity using fluorescence detection, as shown in Figure 15.

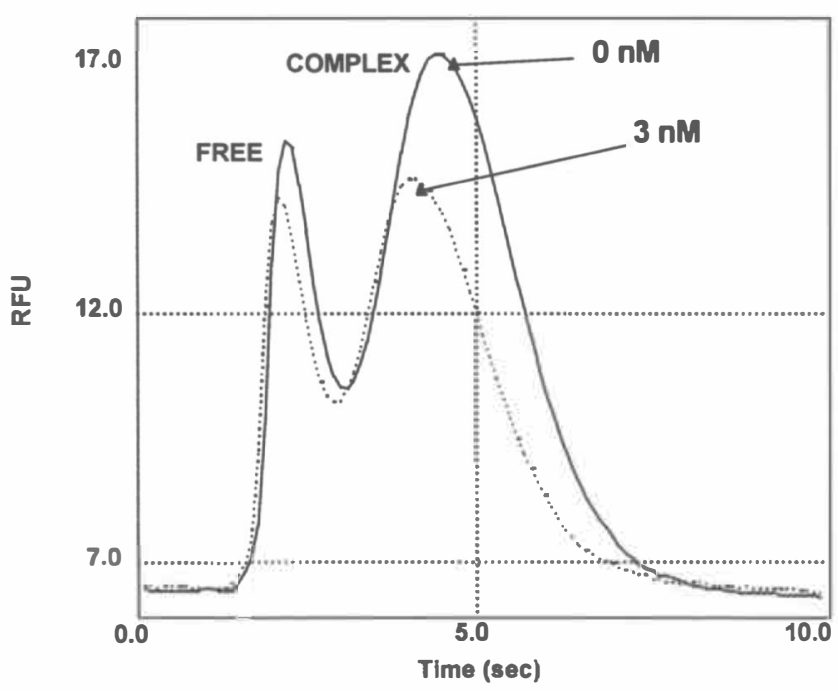

Figure 15. Competitive immunoassay of human insulin standards on a plastic chip; Displacement of CY-5 insulin from complex by human insulin. Effective separation distance: $1.0 \mathrm{~mm}$.

In the case of enzyme assays, we have demonstrated electroosmotic pumping of enzyme, inhibitor and substrate through plastic microchannels, on-chip enzymatic reaction, with no inhibition from the plastic, and separation of peptide substrate from product. Representative results from such an assay are shown in Figure 16.
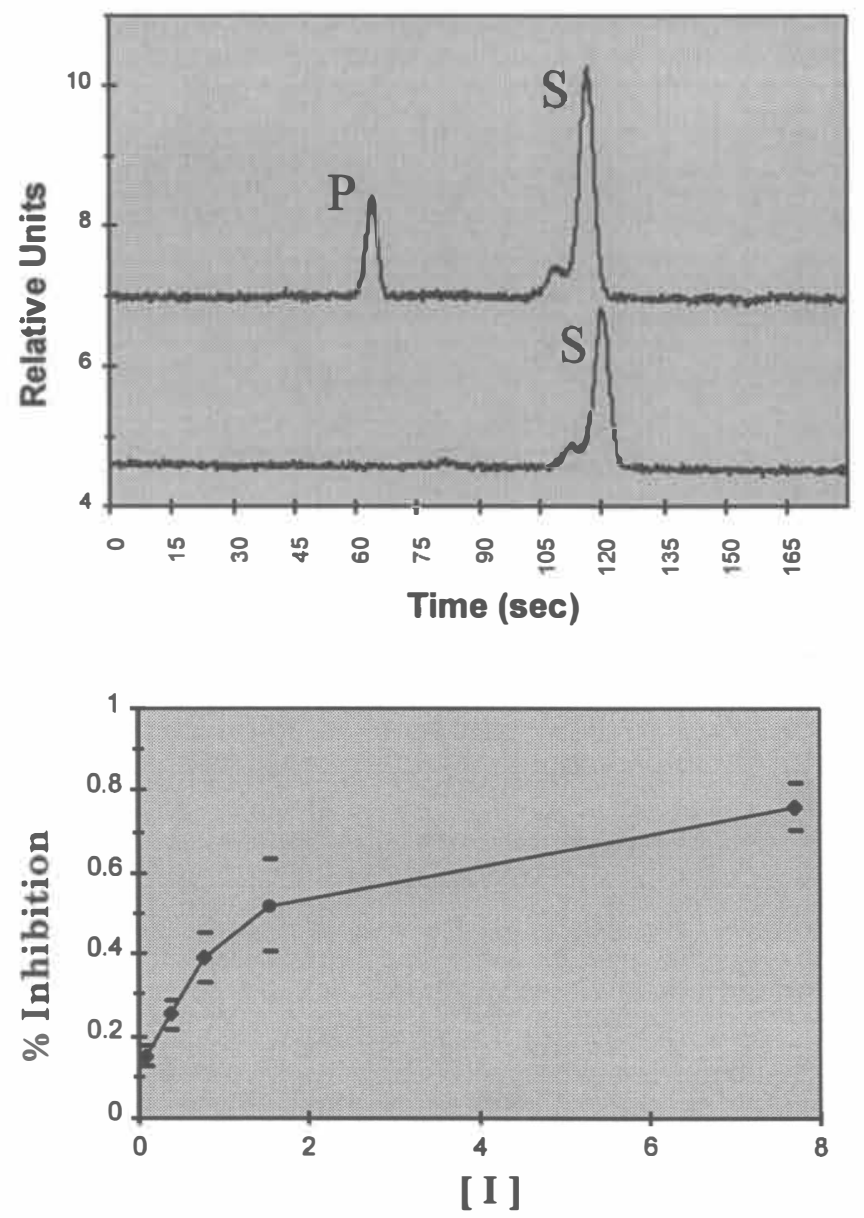

Figure 16. Enzyme inhibition assay. Top: Separations of peptide substrate $(S)$ and product $(P)$ resulting from an enzymatic reaction. Bottom: An IC50 curve generated from running the enzymatic assay with varying levels of a test inhibitor.

\section{Microbead Manipulation}

In addition to solution-phase assays, SBio has demonstrated the feasibility of manipulating paramagnetic particles in microfluidic devices by electrokinetic and magnetic forces. The particles provide a mobile, and spatially-definable solid phase for use in various assays. An injection of particles at a cross-channel is shown in Figure 17. Plugs of magnetic microbeads can be captured with a magnet in a well-defined zone downstream of the injection point, as shown in Figure 18. Beads can be released by subsequent removal of the magnetic field.
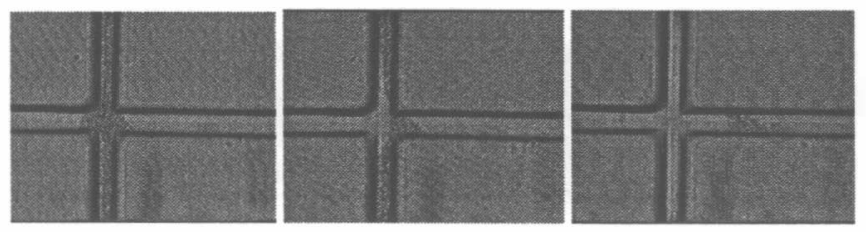

Figure 17. Snapshots (taken at $\sim 0.1 \mathrm{sec}$ intervals) of an electrokinetically-injected plug of microbeads in buffer on a plastic chip. . Beads: I 1 m diameter, in $0.1 \%$ suspension in $I X$ $T B E$. 


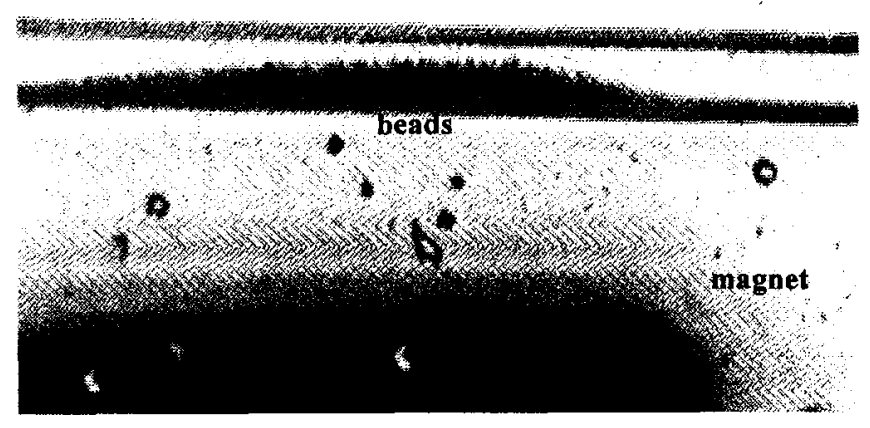

Figure 18. Capture of magnetic beads in a flow field on a plastic chip microchannel of $\sim 100 \mu \mathrm{m}$ width and $\sim 35 \mu \mathrm{m}$ depth. The stream is flowing to the left at $\sim 100 \mu \mathrm{m} / \mathrm{sec}$.

\section{CONCLUSIONS}

We have developed high-volume fabrication methods, surface treatments, and assay protocols to perform rapid biochemical assays on disposable plastic chips. Plastic microfluidic devices offer several advantages over glass or silicon structures including lower processing temperatures, greater surface treatment variety for electro-osmotic flow control, lower cost, and simpler extension to multi-layer device fabrication. Mass-produced, disposable, microfluidic devices hold the promise of revolutionizing drug discovery and diagnostic markets.

\section{REFERENCES}

1. Terry, S.C.; Jerman, J.H.; Angell, J.B. IEEE Trans. Electron. Devices, ED-26, 1880-1886 (1979).

2. Pace, S.J. U.S. Patent 4,908,112, 1990.

3. Kovacs, G.T.A.; Holland, K.O. EP 0376611A2, 1990.

4. Harrison, D.J.; Manz, A.; Fan, Z.; Lüdi, H.; Widmer, H.M. Anal. Chem. 1992, 64, 1926-1932.

5. Effenhauser, C.S.; Manz, A.; Widmer, H.M. Anal. Chem. 1993, 65, 2637-2642.

6. Effenhauser, C.S.; Paulus, A.; Manz, A.; Widmer, H.M.. Anal. Chem. 1994, 66, 2949-2953.

7. Seiler, K.; Fan, Z.H.; Fluri, K.; Harrison, D.J. Anal. Chem. 1994, 66, 3485-3491.

8. Fan, Z.; Harrison, D.J. Anal. Chem. 66, 177-184 (1994).

9. Chiem, N.; Harrison, D.J. Anal. Chem. 1997, 69, 373-378.

10. Jacobson, S.C.; Hergenröder, R.; Moore, Jr., A.W.; Ramsey, J.M. Anal. Chem. 1994, 66, 4127-4132.

11. Jacobson, S.C.; Ramsey, J.M. Anal. Chem. 1996, 68, 720723.

12. Woolley, A.T.; Mathies, R.A. Proc. Natl. Acad. Sci. USA 1994, 91, $11348-11352$.

13. Woolley, A.T.; Mathies, R.A. Anal. Chem. 1995, 67, 3676-3680.

14. Koutny, L.B.; Schmalzing, D.; Taylor, T.A.; Fuchs, M. Anal. Chem. 1996, 68, 18-22.

15. Hadd, A.G.; Raymond, D.E.; Halliwell, J.W.; Jacobson, S.C.; Ramsey, J.M. Anal. Chem. 1997, 69, 3407-3412.

16. Li, P.C.H; Harrison, D.J. Anal. Chem. 69, 1564-1568 (1997).

17. Effenhauser, C.S.; Briun, G.J.M; Paulus, A. Electrophoresis 1997, 18, 2203-2213.
18. Soane, D.S.; Soane, Z.M. US Patent 5,216,022 (1992).

19. Eckström, B.; Jacobson, G.; Ohman, O.; Sjodin, H. PCT Patent Application WO 91/16966 (1991).

20. Effenhauser, C.S.; Bruin, G.J.M.; Paulus, A.; Ehrat, M. Anal. Chem. 69, 3451-3457 (1997).

21. McCormick, R.M.; Nelson, R.J.; Alonso-Amigo, M.G.; Benvegnu, D.J.; Hooper, H.H. Anal Chem. 1997, 69, 26262630.

22. Soane, D.S.; Soane, Z.M.; Hooper, H.H.; Alonso-Amigo, M.G.; PCT Patent Application WO 97/38300 (1997).

23. Huang, X.C.; Quesada, M.A.; Mathies, R.A. Anal. Chem. 1992, 64, 2149-2154.

\section{ACKNOWLEDGEMENTS}

We would like to thank the following researchers at Soane BioSciences for efforts on plastic chip fabrication, surface treatments, DNA separations, immunoassays, enzymatic assays, and microbead manipulation: Goretty Alonso-Amigo, Dominic Benvegnu, Tor Bjornson, Kejun Fan, Danny Hion, Thu Le, Randy McCormick, Alex Sassi, Larry Shea, Anne Wainright, and Qifeng Xue. 